\title{
Reconstrução bilateral de ATM anquilosada por meio de prótese articular: relato de caso
}

\section{Reconstruction of bilateral ankylosed TMJ by prosthetic joint: case report}

\author{
Miguel Scheffer \\ Renato Schroder dos Santos* \\ Pedro Antonio González Hernandez ${ }^{* * *}$ \\ Aurelício Novaes da Silva Júnior ${ }^{* * * *}$
}

\section{Resumo}

Introdução: a anquilose da articulação temporomandibular (ATM) é caracterizada pela fusão intracapsular do complexo disco-côndilo à superfície articular do osso temporal, incluindo adesão fibrosa entre fossa glenoide e eminência articular, o que resulta em restrição dos movimentos mandibulares. O tratamento dessa enfermidade envolve desde a interposição de retalho de músculo temporal até a inserção de próteses articulares. Relato do caso: os autores apresentam um caso de anquilose bilateral de ATM tratado com prótese total, evidenciando seus resultados. Considerações finais: a substituição total da ATM por implante aloplástico pode promover benefícios funcionais não somente para pacientes com anquilose como também para aqueles que possuem malformação congênita envolvendo essa estrutura.

Palavras-chave: Transtornos da articulação temporomandibular. Cirurgia. Anquilose. Desenho de prótese.

\section{Introdução}

A anquilose da articulação temporomandibular (ATM) é um grande problema dentro da cirurgia oral e maxilofacial, sendo o seu tratamento um grande desafio. Dependendo da idade de incidência e do tempo de anquilose, os pacientes apresentam vários sinais clínicos, incluindo: incapacidade de abrir a boca, assimetria facial, micrognatia mandibular e eixo transverso do plano oclusal alterado ${ }^{1,2}$.

O diagnóstico pode ser complementado por radiografias extraorais (panorâmica, submentovertex e incidência de Waters). A tomografia computadorizada promove uma imagem de qualidade melhor, particularmente quando reconstruções multiplanas sagitais estão disponíveis.

Mestre em Cirurgia e Traumatologia Bucomaxilofacial, doutorando em Odontologia pela Ulbra de Canoas, Porto Alegre, RS, Brasil. Mestre em Cirurgia e Traumatologia Bucomaxilofacial pela PUCRS, Porto Alegre, RS, Brasil.

Mestre em Cirurgia e Traumatologia Bucomaxilofacial (Faculdade de Odontologia da Universidade Federal de Pelotas), doutor em Dentística Restauradora (Universidade Estadual Paulista Júlio de Mesquita Filho), professor adjunto do curso de Graduação da Ulbra de Canoas, pró-reitor adjunto de Graduação da Ulbra de Canoas.

*** Mestre e doutor em Cirurgia e Traumatologia Bucomaxilofacial pela PUCRS, Porto Alegre, RS, Brasil, professor adjunto da Ulbra, Canoas, RS, Brasil. 
Para o tratamento da anquilose da ATM, muitos métodos têm sido usados, desde a liberação da anquilose, passando pela inserção de material interposicional, até a completa reconstrução da articulação $0^{3,4}$. Na reconstrução das articulações, incluindo a articulação temporomandibular, as técnicas e os materiais que promovem os melhores resultados para os pacientes têm sido amplamente debatidos ${ }^{5}$.

Podem beneficiar-se da reconstrução dessa articulação, utilizando uma prótese total, pacientes com condições específicas e patologia na ATM, incluindo: múltiplas cirurgias na ATM (duas ou mais cirurgias prévias); implantes aloplásticos prévios, entre os quais Proplast-Teflon (Vitek, Inc., Houston, TX, USA) e silicone (Dow-Corning, Midland, MS, USA); osteoartrite com comprometimento do côndilo e fossa; patologia inflamatória ou reabsortiva da ATM; doenças do tecido conjuntivo ou autoimunes afetando a ATM (ex.: artrite psoriática, espondilite ancilosante, esclerodermia, síndrome de Sjogren's, artrite reumatoide, lúpus, etc.); anquilose fibrosa ou óssea; estruturas anatômicas ausentes ou deformadas (isto é, côndilos deslocados fraturados, ausência de côndilos e porções do ramo como resultado de trauma anterior, cirurgia, patologia ou deformidade congênita); e tumores envolvendo a articulação e área mandibular. Essas condições podem criar uma disfunção e/ou destruição da articulação do côndilo mandibular até a fossa glenoide ${ }^{6}$.

O objetivo deste artigo é relatar o tratamento de um caso de anquilose bilateral de ATM por meio da instalação de duas próteses totais de ATM.

\section{Relato do caso}

Paciente do sexo feminino, 30 anos, solteira, apresentou-se com queixa de limitação de abertura bucal e dores nas ATMs direita e esquerda. Relatou, ainda, que havia sofrido um acidente automobilístico há três anos, sendo tratada com bloqueio maxilomandibular por 45 dias. Além disso, não conseguia executar movimentos de mastigação e fonação adequadamente. Utilizava medicações analgésicas como paracetamol gotas diariamente para controle da dor e antidepressivos para controle da ansiedade. Foi submetida a artrocentese bilateral sem obter melhora de sua abertura bucal. Além desses tratamentos, a paciente referiu ter sido tratada com artroplastia do côndilo com interposição de fáscia do músculo temporal. Os resultados desses tratamentos não foram eficazes. Ao exame clínico, comprovou-se importante limitação da abertura bucal (4 mm) (Fig. 1) e, ao exame tomográfico (Fig. 2), observou-se nítida presença de anquilose óssea da ATM. Diante desse quadro, optou-se pela reconstrução articular com prótese total de ATM. Foi realizado tratamento cirúrgico sob anestesia geral.

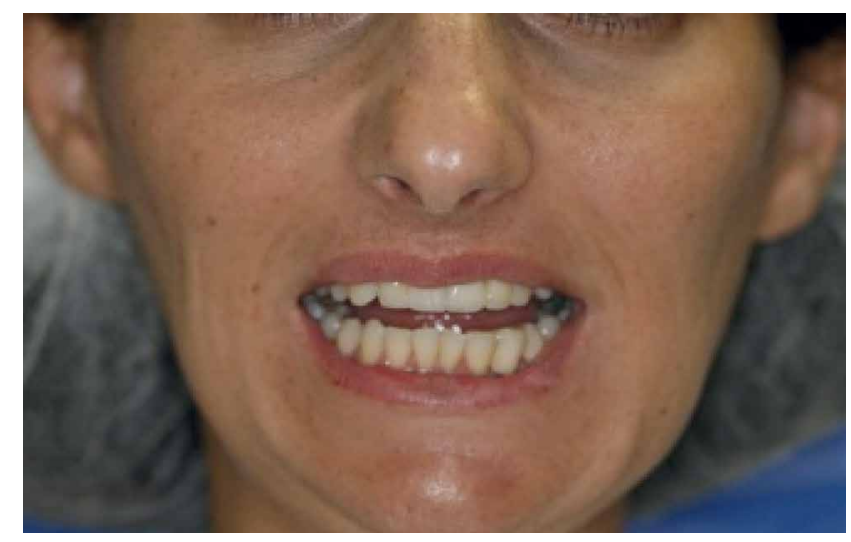

Figura 1 - Limitação da abertura bucal

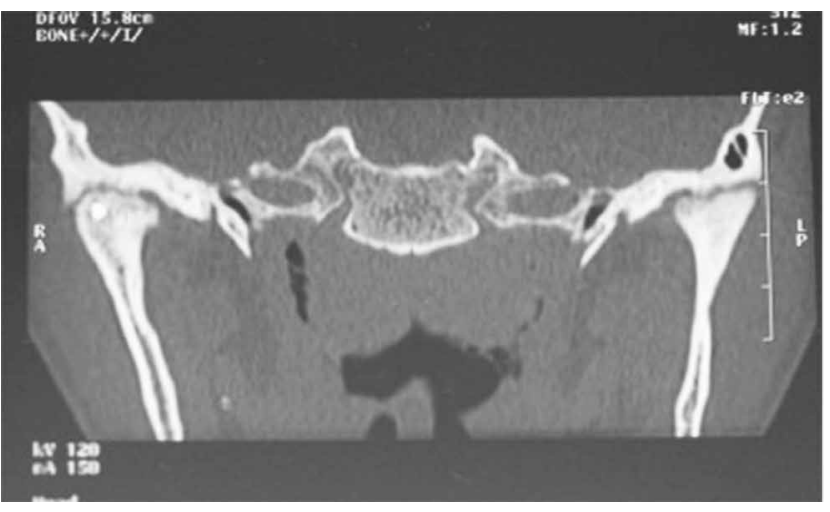

Figura 2 - Exame tomográfico

Efetuou-se a remoção da anquilose bilateral através de incisão pré-auricular (Fig. 3) direita e esquerda e instalação de componente protético de fos-

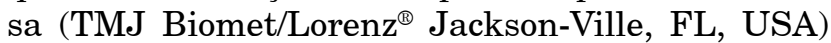
no arco zigomático (Fig. 4). Além disso, efetuou-se acesso submandibular (Fig. 5) para instalação e fixação de componente protético de ramo mandibular direito e esquerdo (TMJ Biomet/Lorenz ${ }^{\circledR}$ Jackson-Ville, FL, USA). Acrescentou-se, também, gordura removida, por cirurgião plástico, da região abdominal (Fig. 6) sobre a interface côndilo fossa, na tentativa de evitar formação de células gigantes. A síntese foi feita em dois planos com Monocryl ${ }^{\circledR}$ 4-0 (Ethicon, Johnson-Johnson, Cincinnati, Ohio, USA) e Mononylon ${ }^{\circledR}$ 6-0 (Ethicon, Johnson-Johnson, Cincinnati, Ohio, USA). Utilizou-se Tylex ${ }^{\circledR} 30 \mathrm{mg}$ por uma semana para controle da dor, e fisioterapia da ATM foi estabelecida após 30 dias pelo período de três meses para melhorar a abertura bucal. A paciente evoluiu sem complicações, realizou 20 reconsultas e relatou satisfação com os resultados atingidos. Após três anos de acompanhamento, evidenciou-se aumento da abertura bucal (Fig. 7), ausência de dores e melhora do quadro nutricional, bem como mastigação e fonação. A imagem da radiografia panorâmica sugere boa adaptação da prótese articular (Fig. 8). Cabe ressaltar, ainda, que a paciente assinou um termo de consentimento autorizando a publicação dos dados. 


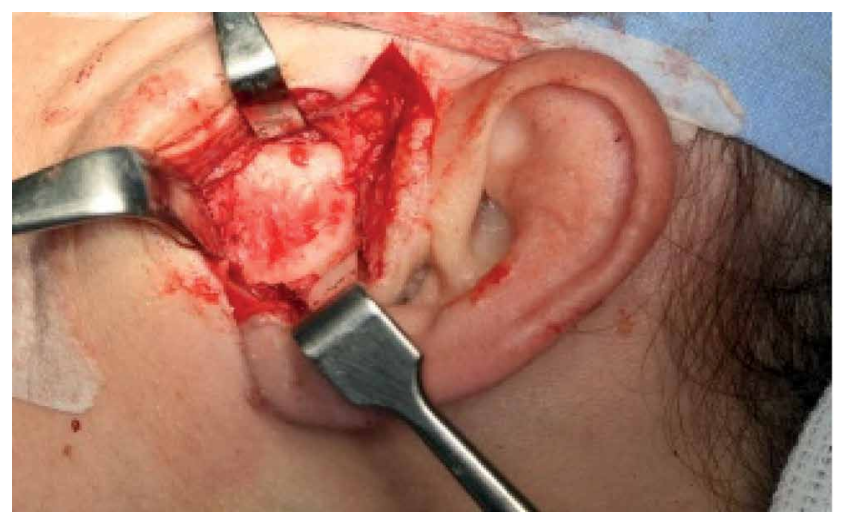

Figura 3 - Incisão pré-auricular

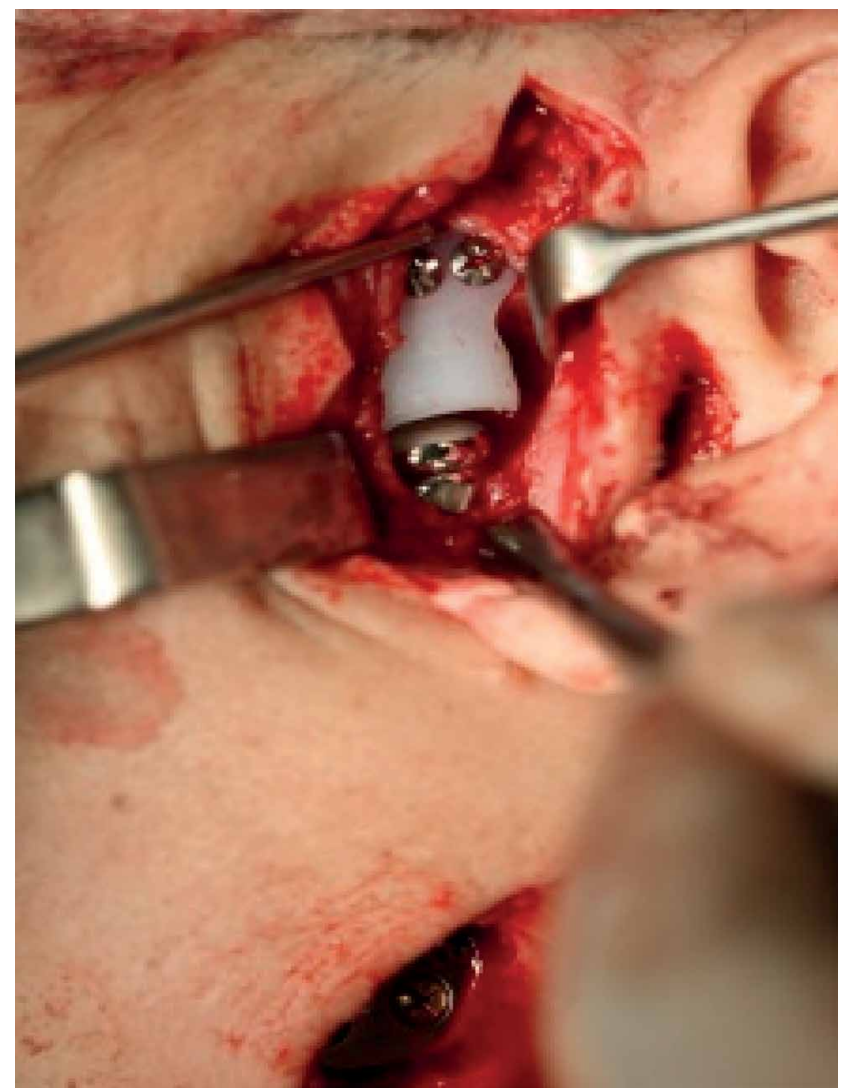

Figura 4 - Instalação de componente protético de fossa

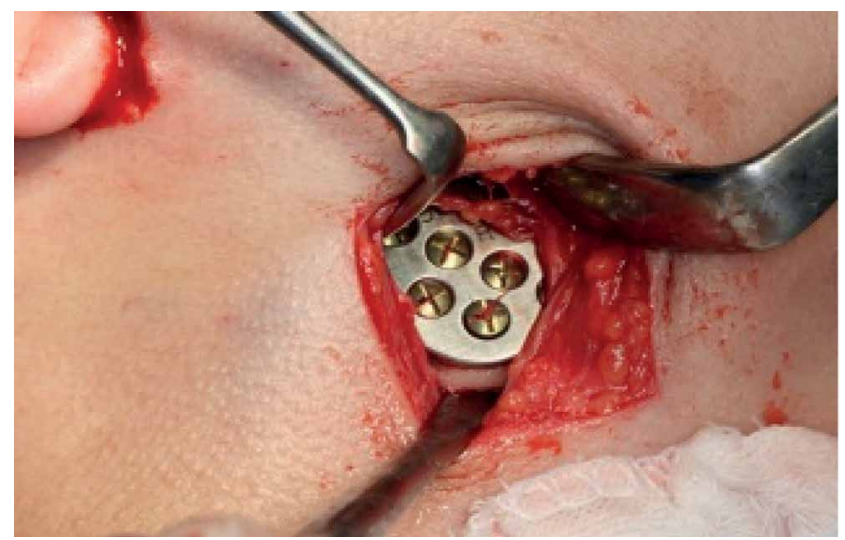

Figura 5 - Acesso submandibular

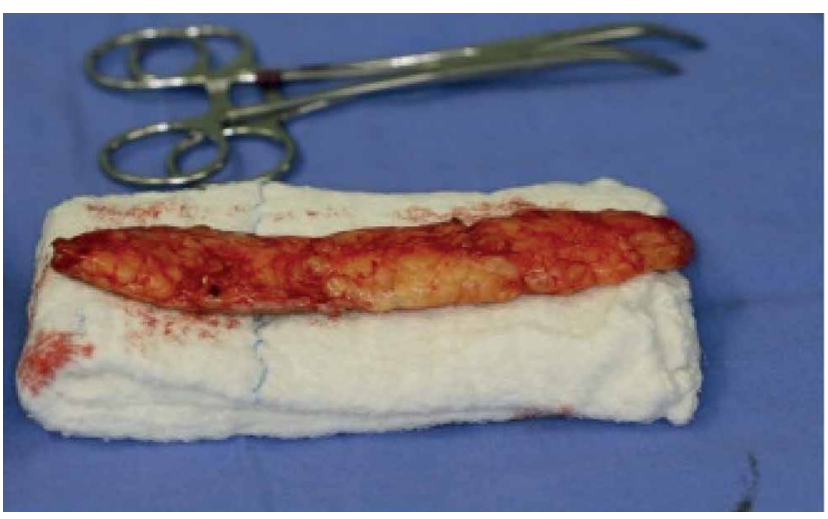

Figura 6 - Gordura abdominal

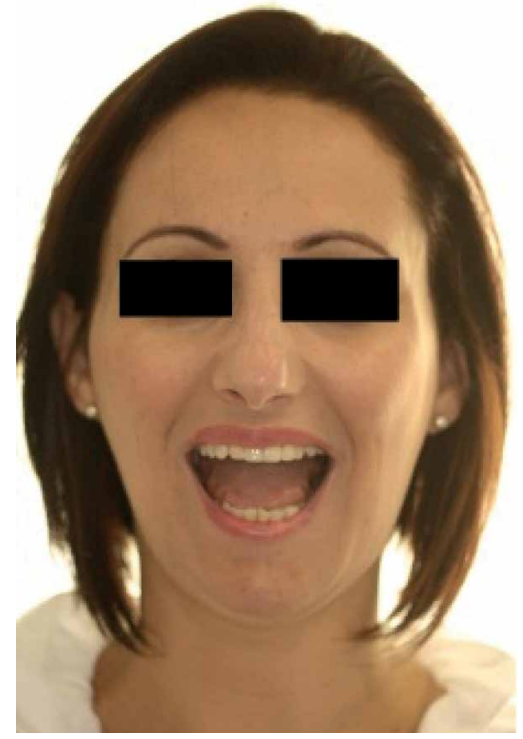

Figura 7 - Aumento da abertura bucal após cirurgia

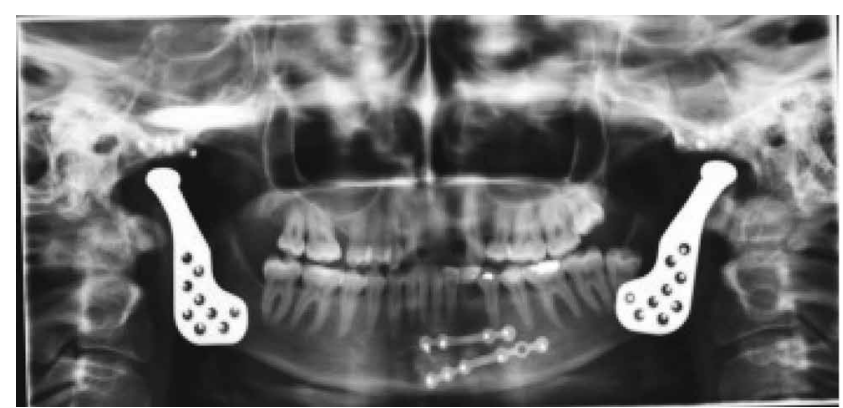

Figura 8 - Radiografia panorâmica pós-operatória

\section{Discussão}

Estima-se que as doenças da ATM afetem aproximadamente 30 milhões de norte-americanos, com uma estatística de um milhão de novos pacientes diagnosticados anualmente ${ }^{7,8}$. Embora a maioria desses pacientes possa ser tratada com terapias não cirúrgicas, ainda permanece um grupo que necessita obrigatoriamente da intervenção cirúrgica. Quando uma intervenção cirúrgica da ATM a céu 
aberto é necessária, essa estrutura pode ser reparada ou reconstruída com tecido autógeno ou por meio de implantes aloplásticos ${ }^{9}$.

A anquilose da articulação temporomandibular pode ser classificada em anquilose verdadeira (intracapsular) ou pseudoanquilose (extracapsular). A anquilose verdadeira da articulação pode ser definida como qualquer condição que produza adesões fibrosas ou ósseas na superfície da ATM e é comumente decorrente de trauma ou infecção. No entanto, podem também estar associadas a artrite reumatoide, neoplasias, complicações cirúrgicas locais e extensão da anquilose intracapsular. Já a pseudoanquilose resulta de desordens musculares, ósseas, neurológicas ou psiquiátricas ${ }^{10,11}$.

O manejo da anquilose verdadeira implica a intervenção cirúrgica. A necessidade de usar um material interposicional para prevenir a reanquilose após o tratamento de artroplastia da ATM tem sido muito discutida ${ }^{12,13}$. Uma variedade de materiais interposicionais tem sido usada, incluindo fáscia do músculo temporal, derme, cartilagem auricular, fáscia lata, gordura, silicone, entre outros ${ }^{14}$.

Herbosa e Rotskoff ${ }^{15}$ (1990) observaram 15 pacientes com problemas de ATM no pré e pós-operatório e avaliaram a eficácia do composto (fáscia, músculo, periósteo) do retalho de pedículo de músculo temporal como substituto do disco interposicional. Oito meses depois da cirurgia verificou-se melhora clínica significativa no movimento mandibular ( $p$ $<0,05)$. Entretanto, uma significativa redução da translação $(p<0,01)$ foi evidente, indicando que $o$ aumento da abertura mandibular foi possível graças ao movimento compensatório rotacional. Este estudo demonstrou que o retalho de músculo temporal é um bom tecido autógeno para reconstrução da ATM.

Matsuura et al. ${ }^{16}$ (2001) estudaram as mudanças anatômicas e funcionais depois de realizar artroplastia sem interposição de material no espaço entre côndilo e fossa mandibular. Usando modelos animais, os autores mostraram que essa técnica foi incapaz de restaurar a função normal da ATM no tratamento de anquilose.

De acordo com seus estudos, Elgazzar et al. ${ }^{17}$ (2010) concluíram que a liberação antecipada da ATM anquilosada com reconstrução do ramo através de distração osteogênica ou artroplastia com enxerto ósseo, seguido de vigorosa fisioterapia, é uma boa alternativa para gestão da ATM anquilosada. Apesar de potenciais problemas relacionados com enxerto costocondral em estudos anteriores, os autores indicam essa alternativa de tratamento para pacientes em crescimento.

Westermark $^{18}$ (2010), após oito anos de controle pós-operatório de 12 pacientes que foram submetidos à prótese da ATM, constatou que a média da capacidade de abertura bucal, que era de $3,8 \mathrm{~mm}$ antes do procedimento, passou para $30,2 \mathrm{~mm}$. Todos relataram ausência de dores e tiveram melhora na mastigação. Não houve lesão permanente do nervo facial, e nenhum paciente apresentou infecção.

A história da reconstrução aloplástica da ATM tem sido caracterizada por muitas falhas, ocasionadas por formato inapropriado, falta de atenção nos princípios biomecânicos e ignorância quanto ao que já foi documentado na literatura ortopédica, principalmente em relação à fadiga e quebra de próteses com encaixe metal com metal ${ }^{19}$.

Saeed et al. ${ }^{20}$ (2002) estudaram 56 pacientes com 76 reconstruções costocondrais da ATM e concluíram que, embora o enxerto costocondral funcione bem em muitas situações, um diagnóstico pré-operatório de anquilose estava associado a um alto índice de complicações e altas taxas de novas cirurgias, sugerindo precauções nesse grupo de pacientes. Em estudo multicêntrico foram comparados os resultados de 49 pacientes com enxerto costocondral e 50 pacientes com reconstrução aloplástica com prótese de ATM Christensen ${ }^{\circledR}$ (TMJ Inc, Golden, CO, USA). Verificou-se melhora nos sintomas dos pacientes de ambos os grupos, porém a recidiva de anquilose necessitando de novo procedimento cirúrgico foi mais presente no grupo tratado com enxerto. Dezoito pacientes do grupo costocondral desenvolveram anquilose, enquanto nenhum paciente do grupo da prótese aquilosou. Isso mostra um grande risco de reanquilose com a utilização de enxertos ósseos.

Muitos pacientes que são candidatos à reconstrução da ATM tiveram intervenções prévias. As cicatrizes decorrentes dos procedimentos prévios podem resultar em maior dificuldade de dissecção e aumentam o risco de dano ao nervo facial. Alguns relatos sugerem que o sucesso do tratamento de reconstrução protética da ATM está inversamente relacionado com o número de procedimentos cirúrgicos prévios ${ }^{21}$.

As articulações temporomandibulares complexas e/ou com múltiplas cirurgias apresentam-se como grandes desafios em relação ao manejo e à previsibilidade dos tratamentos ${ }^{7}$. Esse tipo de paciente talvez não tenha somente uma patologia significante da ATM, mas também frequentemente dor severa associada à deformidade facial, muscular e de maloclusão.

\section{Considerações finais}

Embora o tratamento da anquilose tenha sido discutido na literatura por muito tempo, nenhuma técnica isolada tem produzido resultados satisfatórios. Pela literatura consultada e pelo caso relatado neste estudo, pode-se concluir que a técnica de substituição por prótese mostrou-se importante alternativa no tratamento de anquilose da ATM, principalmente por apresentar baixa incidência de rean- 
quilose e devolver equilíbrio mastigatório. Apesar disso, estudos clínicos prospectivos em longo prazo e revisões sistemáticas dessa técnica cirúrgica ainda se fazem necessários para sua indicação segura no tratamento da anquilose da ATM.

\section{Abstract}

Introduction: ankylosis of the temporomandibular joint (TMJ) is characterized by the intracapsular fusion of the complex condyle disc to the joint surface of the temporal bone, including fibrous adhesions between glenoid fossa and joint eminence, which results in the restriction of mandibular movements. The treatment of this condition involves from the interposition of temporalis muscle flap to the insertion of joint prostheses. Case report: the authors present a case of bilateral TMJ ankylosis treated with total joint prosthesis, showing its results. Final considerations: total TMJ replacement by alloplastic implant may promote functional benefits not only for patients with ankylosis, but also for those who have congenital malformations involving this structure.

Keywords: Temporomandibular joint disorders. Surgery. Ankylosis. Prosthesis design.

\section{Referências}

1. El-Sheikh MM. Temporomandibular joint ankylosis the Egyptian experience. Ann Roy Coll Surg Engl 1999; 81:12-8.

2. Macintosh RB. The use of autogenous tissues for temporomandibular joint reconstruction. J Oral Maxillofacial Surg 2000; 58:63-9.

3. Chen C, Lai J, Chen Y. Costocondral graft in acute mandibular condylar fracture. Plast Reconstruct Surg 1997; 100:1234-9.

4. Chossegros C, Guyot L, Cheynet F, Blanc JI, Gola R, Bourezak Z, et al. Comparison of different material for interposition arthroplasty in treatment of temporomandibular joint ankylosis surgery: long- term follow-up in 25 cases. Br J Oral Maxillofac Surg 1997; 35:157-60.

5. Wolford L. Factors to consider in joint prosthesis systems. Proc (Bayl Univ Med Cent) 2006; 19:232-8.

6. Wolford L, Dingwerth DJ, Talwar RM, Pitta MC. Comparison of 2 temporomandibular joint total joint prosthesis system. J Oral Maxillofacial Surg 2003; 61:685-90.

7. Wolford L, Cottrell D, Henry C. Temporomandibular joint reconstruction of complex patient with the techmedica custom-made total joint prosthesis. J Oral Maxillofac Surg $1994 ; 52: 2-10$.

8. Baird DN, Rea WJ. The temporomandibular joint implant controversy: a review of autogenous/alloplastic materials and their complications. J Nutr Environ Med 1998; 8:289-300.

9. Stevão E, Stevão K. Tratamento ortopédico para a articulação temporomandibular. J Bras Oclusão, ATM e Dor Orofacial 2005; 5(18):32-42.
10. Bergey DA, Braun TW. The posterior zygomatic arch osteotomy to facilitate temporalis flap placement. J Oral Maxillofac Surg 1994; 52(4):426-7.

11. Faerger TH, Ennis RL, Allen GA. Temporomandibular joint ankylosis following mastoiditis: report of a case. J Oral Maxillofac Surg 1990; 48(8):866-70.

12. Hebel G, Hensher R. The versatility of the temporalis muscle flap in reconstructive surgery. Br J Oral Maxillofac Surg 1986; 24(2):96-101.

13. Haidar Z. Ankylosis of the temporomandibular joint: causes and management. J Oral Med 1986; 41(4):246-9.

14. Omura S, Fujita K. Modification of the temporalis muscle and fascia flap for the management of ankylosis of the temporomandibular joint. J Oral Maxillofac Surg 1996; 54(6):794-5.

15. Herbosa EG, Rotskoff KS. Composite temporalis pedicle flap as an inter positional graft in temporomandibular joint arthroplasty: a preliminary report. J Oral Maxillofac Surg 1990; 48(10):1049-56.

16. Marsuura H, Miyamoto H, Ogi N, Kurita K, Goss AN. The effect of gap arthroplasty on temporomandibular joint ankylosis: an experimental study. Int J Oral Maxillofac Surg 2001; 30:431-7.

17. Elgazzar RF, Abdelhady AI, Saad KA, Elshaal MA, Hussain MM, Abdelal SE, et al. Treatment modalities of TMJ ankylosis: experience in DeltaNile, Egypt. Int J Oral Maxillofac Surg 2010; 39:333-42.

18. Westermark A. Total reconstruction of the temporomandibular joint. Up to 8 years of follow-up of patients treated with Biomet/Lorenz total joint prostheses. Int J Oral Maxillofac Surg 2010; 39:951-5.

19. Quinn P. Total temporomandibular joint reconstruction. Oral and Maxillofacial Surg Clin North Am, 2000; 12:93-104.

20. Saeed NR, Hensher R, McLeod NMH, Kent JN. Reconstruction of the temporomandibular joint autogenous compared with alloplastic. Br J Oral Maxillofac Surg 2002; 40:296-9.

21. Wolford LM, Pitta MC, Reiche-Fishel O, Franco PF. TMJ Concepts/Techmedica custom-made TMJ total joint prosthesis: 5 year follow-up study. Int J Oral Maxillofac Surg 2003; $32: 268-74$

\section{Endereço para correspondência:}

Renato Schroder dos Santos

Rua Mostardeiro, 1000 / 301 - Bairro Independência 90430-000 Porto Alegre/RS

Fone: (51) 85619766

E-mail: renatoss10@yahoo.com.br

Recebido: 19/03/2013. Aceito: 13/11/2013. 